\title{
Stress can attenuate hepatic lipid accumulation via elevation of hepatic 6 -muricholic acid levels in mice with nonalcoholic steatohepatitis
}

Sayuri Takada, Tsutomu Matsubara, Hideki Fujii, Misako Sato-Matsubara, Atsuko Daikoku, Naoshi Odagiri, Yuga Amano-Teranishi, Norifumi Kawada, Kazuo Ikeda

\begin{tabular}{|c|l|}
\hline Citation & Laboratory Investigation. 101; 193-203 \\
\hline Issue Date & $2021-02$ \\
\hline Published & 2020-12-10 \\
\hline Type & Journal Article \\
\hline Textversion & Author \\
\hline $\begin{array}{c}\text { Supplementary } \\
\text { information }\end{array}$ & $\begin{array}{l}\text { Supplementary materials is available online at } \\
\text { https://doi.org/10.1038/s41374-020-00509-x. }\end{array}$ \\
\hline Rights & $\begin{array}{l}\text { The following article has been accepted by Laboratory Investigation. This is the } \\
\text { accepted manuscript version. The final, published version is available at } \\
\text { https://doi.org/10.1038/s41374-020-00509-x }\end{array}$ \\
\hline DOI & 10.1038/s41374-020-00509-x \\
\hline
\end{tabular}

Self-Archiving by Author(s) Placed on: Osaka City University Repository 
Stress can attenuate hepatic lipid accumulation via elevation of hepatic $\beta$-muricholic acid levels in mice with non-alcoholic steatohepatitis.

Sayuri Takada ${ }^{1,2}$, Tsutomu Matsubara ${ }^{1, *}$, Hideki Fujiii, ${ }^{2,3}$, Misako Sato-Matsubara ${ }^{2,4}$, Atsuko Daikoku $^{2}$, Naoshi Odagiri ${ }^{2}$, Yuga Amano-Teranishi ${ }^{2}$, Norifumi Kawada ${ }^{2}$, Kazuo Ikeda $^{1}$

1. Department of Anatomy and Regenerative Biology, Osaka City University Graduate School of Medicine, Osaka, Japan.

2. Department of Hepatology, Osaka City University Graduate School of Medicine, Osaka, Japan.

3. Endowed Department of Liver Cirrhosis Therapeutics, Osaka City University Graduate School of Medicine, Osaka, Japan.

4. Endowed Laboratory of Synthetic Biology, Osaka City University Graduate School of Medicine, Osaka, Japan.

*Correspondence: Tsutomu Matsubara, Department of Anatomy and Regenerative Biology, Osaka City University Graduate School of Medicine. 1-4-3 Asahimachi, Abeno, Osaka 5458585, Japan. Tel: +81-6-6645-3701, Fax: +81-6-6645-3702, Email: matsu335@med.osakacu.ac.jp.

Running title: Influence of stress in NAFLD 


\section{Abstract}

Stress can affect our body and is known to lead to some diseases. However, the influence on the development of non-alcohol fatty liver disease (NAFLD) remains unknown. This study demonstrated that chronic restraint stress attenuated hepatic lipid accumulation via elevation of hepatic $\beta$-muricholic acid ( $\beta$ MCA) levels in the development of non-alcoholic steatohepatitis (NASH) in mice. Serum cortisol and corticosterone levels, i.e., human and rodent stress markers, were correlated with serum bile acid levels in patients with NAFLD and methionine- and choline-deficient (MCD) diet-induced mice, respectively, suggesting that stress is related to bile acid (BA) homeostasis in NASH. In the mouse model, hepatic $\beta$ MCA and cholic acid (CA) levels were increased after the stress challenge. Considering that a short stress enhanced hepatic CYP7A1 protein levels in normal mice and corticosterone increased CYP7A1 protein levels in primary mouse hepatocytes, the enhanced Cyp7al expression was postulated to be involved in the chronic stress-increased hepatic $\beta \mathrm{MCA}$ level. Interestingly, chronic stress decreased hepatic lipid levels in MCD-induced NASH mice. Furthermore, $\beta \mathrm{MCA}$ suppressed lipid accumulation in mouse primary hepatocytes exposed to palmitic acid/oleic acid, but CA did not. In addition, Cyp7al expression seemed to be related to lipid accumulation in hepatocytes. In conclusion, chronic stress can change hepatic lipid accumulation in NASH mice, disrupting BA homeostasis via induction of hepatic Cyp7al expression. This study discovered a new $\beta$ MCA action in the liver, indicating the possibility that $\beta \mathrm{MCA}$ is available for NAFLD therapy. 
Abbreviations: ABC, ATP-binding cassette; ALT, alanine aminotransferase; BA, bile acid; BAAT, bile acid-CoA:amino acid $\mathrm{N}$-acyltransferase; $\beta \mathrm{MCA}$, beta-muricholic acid; CA, cholic acid; CYP, cytochrome P450 family; CV, central vein; FXR, farnesoid X receptor; HE, haematoxylin and eosin; HNF4A, hepatic nuclear factor 4 alpha; LRH1, liver rich homologue 1; MCD, methionine- and choline-deficient; MCS, methionine- and choline-supplemented; NAFLD, non-alcoholic fatty liver disease; NASH, non-alcoholic steatohepatitis; NEFA, nonesterified fatty acid; OA, oleic acid; PA, palmitic acid; PV, portal vein; SLC, solute carrier family; SHP, small heterodimer partner; TChol, total cholesterol; T $\beta$ MCA, tauro-betamuricholic acid; TCDCA, taurochenodeoxycholic acid; TDCA, taurodeoxycholic acid; TG, triglyceride. 


\section{Introduction}

Non-alcoholic fatty liver disease (NAFLD) is a disease that causes fat deposition in the liver and occurs in people with alcohol consumption of less than $30 \mathrm{~g} / \mathrm{day}$ for males and 20 g/day for females in terms of ethanol [1]. NAFLD is classified into simple steatosis and steatohepatitis by pathological diagnosis. The progressive type of NAFLD, which is called nonalcoholic steatohepatitis (NASH), can lead to cirrhosis and hepatocellular carcinoma [2]. Approximately $60 \%$ of patients with NAFLD are estimated to develop NASH [3]. Obesity and diabetes are involved in the onset of NAFLD $[4,5]$, and patients with NAFLD are increasing in many areas of the world and have been estimated to be approximately 1 billion people [6]. Recently, some studies have shown that patients with NASH show a significantly higher mortality rate than those with simple fatty liver and the general population [7]. Hence, elucidating the pathology of NAFLD and identifying its therapeutic targets is critical.

NAFLD is excess lipid accumulation in the liver, resulting from excessive adipose tissue lipolysis and increased liver uptake of free fatty acids, enhanced hepatic lipogenesis and attenuated hepatic lipolysis [8]. Thus, controlling hepatic lipid accumulation could be a therapeutic target for NAFLD. Several studies have shown that proinflammatory cytokines, such as the tumour necrosis factor- $\alpha$, contribute significantly to the progression from steatosis to steatohepatitis [9-11]. Furthermore, these cytokines likely cause changes in endogenous lipid and bile acid metabolite profiles by altering metabolic cascades in the liver [12]. However, the mechanisms underlying these metabolic alterations are not completely understood. Factors other than these cytokines might be involved.

Stress is used to refer to a condition where various or perceived stimuli alter the homeostatic state of an organism [13]. Stress, such as life-changing or threatening events, is 
well known to lead to activation of the hypothalamic-pituitary-adrenal axis and the sympathetic nervous system $[14,15]$. The activation stimulates the release of the important mediators catecholamines and glucocorticoids from the adrenal grands. Several studies have established an increasingly clear link between stress and viral hepatitis, such as the correlation between psychosocial stress and the severity of chronic hepatitis $C[16]$ and the negative effect of chronic psychosocial stress on the antibody response after hepatitis B vaccination [17]. Thus, stress may influence liver diseases via the central nervous system/adrenal gland axis, but the evidence is very poor, especially for the development of NAFLD.

In this study, the influence of restraint stress on the development of NASH was assessed in mice fed a methionine- and choline-deficient (MCD) diet to understand the molecular alterations in our bodies. Our data showed that restraint stress altered hepatic lipid accumulation following elevated hepatic $\beta$-muricholic acid ( $\beta \mathrm{MCA}$ ) levels in MCD dietinduced steatohepatitis. 


\section{Materials and Methods}

\section{Materials}

All reagents were obtained from Merck Co (Kenilworth, NJ, USA) or FUJIFILM Wako Pure Chemical Corporation (Osaka, Japan), unless otherwise noted. Tauro- $\beta$-muricholic acid (T $\beta$ MCA) and $\beta$ MCA were purchased from Cayman Chemical Company (Ann Arbor, MI, USA).

\section{Patients}

The study protocol with patient samples conformed to the ethical guidelines of the Declaration of Helsinki. All patients were informed of the investigational nature of this study and gave their written informed consent. The study was approved by the Ethics Committee of Osaka City University Graduate School of Medicine (Institutional Review Board number 3641). In this study, we diagnosed patients with NAFLD by abdominal ultrasound. Fifty-one patients with NAFLD were studied. Of these, 21 patients were excluded because they were taking some medicine that can change bile acid levels. The clinical information for the patients is summarized in Supplementary Table 1.

\section{Generation of NASH mice and challenge with restraint stress}

Male mice (C57BL/6, 10 weeks old) were purchased from Japan SLC, Inc. (Shizuoka, Japan) and housed in temperature- and light-controlled rooms and given water and CLEA Rodent Diet CE-2 (CLEA Japan, Inc, Tokyo, Japan) as standard chow. One week later, the mice were divided into 4 groups. To generate NASH, two mouse groups were given a 
methionine- and choline-deficient (MCD) diet with 60\% fat (Supplementary Table 2) ad libitum as the MCD-diet group. On the other hand, the other groups were freely given the MCD diet and drinking water supplemented with $4 \mathrm{mg} / \mathrm{mL}$ methionine and $30 \mathrm{mg} / \mathrm{mL}$ choline ad libitum as the control group (MCS-diet group). The mice were exposed to restraint stress 2 hours daily (10:30-11:30 and 16:00-17:00) for 21 days as the stress group. The mice were sacrificed at 18 hours after the final challenge of the stress. Throughout the stress task, individual mice were restricted to adequately ventilated $50-\mathrm{ml}$ conical plastic tubes $(3 \times 10-\mathrm{cm}$ Falcon tubes). The mice could rotate from the supine to prone position, but they could not turn head to tail. The other mice were moved to new cages and undisturbed as the non-stressed control group (non-stress group), but food and water were removed to match the drinking and feeding conditions of the stress group. No change in food and diet consumption was observed between stress and non-stress groups. To investigate the direct effect of stress, another mouse set (C57BL/6 male mice, 10 weeks old) was exposed to restraint stress for 2 days (short stress) under CLEA Rodent Diet CE-2 conditions. The mice were sacrificed at 6 hours after the final stress challenge. The animal studies were carried out in accordance with protocols approved by the Institutional Animal Care and Use Committee of Osaka City University, Osaka.

\section{Serum and liver chemistry}

For the patients, the serum bile acid level was measured by LSI Medience Corporation (Tokyo, Japan). The serum cortisol level was determined by using a Cortisol ELISA Kit (Cayman Chemical). For mice, the serum catalytic activity of alanine aminotransferase (ALT) was measured with an ALT assay kit. The serum corticosterone level was determined by using a Corticosterone ELISA Kit (Cayman Chemical). The liver was homogenized with $50 \mathrm{mM}$ Tris-hydrochloric acid $(\mathrm{HCl})$ buffer supplemented with $0.1 \%$ Triton $\mathrm{X}-100$. The liver homogenate was subjected to bile acid (BA), total cholesterol (TChol), triglyceride (TG), and 
non-esterified fatty acid (NEFA) analysis. The liver and serum BA, TG, TChol, and NEFA levels were estimated with a FUJIFILM Wako Pure Chemical assay kit.

\section{Liquid chromatography-mass spectrometry analysis of bile acid composition}

Liver tissue $(20 \mathrm{mg})$ was homogenized with $600 \mu \mathrm{L}$ of acetonitrile:water (3:2) solution including $5 \mathrm{nM}$ nor-deoxycholic acid (Toronto Research Chemicals, North York, ON, Canada) as an internal standard. The homogenate was centrifuged at $20,000 \mathrm{xg}$ for $10 \mathrm{~min}$, and the supernatant was transferred to a new tube and subjected to a liquid chromatography-mass spectrometry (LC-MS) system for bile acid detection. The LC-MS system consisted of a Nexera X2 LC pump and auto-injector (Shimadzu corporation, Kyoto, Japan) coupled to an LCMS-8060 mass spectrometer (Shimazu). LC-MS was performed based on a method reported previously [18]. Briefly, the samples were separated on an Acquity UPLC BEH C18 (1.7 $\mu \mathrm{m}$, $2.1 \times 100 \mathrm{~mm}$ ) column (Waters corporation, Milford, MA, USA) using the following twentyseven-min elution gradient: the eluent composition was set at $95 \% \mathrm{~A}$ and $5 \% \mathrm{~B}$ during the first 5 min (retention time 0 to $5 \mathrm{~min}$ ) and then linearly changed to $85 \% \mathrm{~A}$ and $15 \% \mathrm{~B}$ in $9 \mathrm{~min}(5$ to $14 \mathrm{~min}$ ); next, the proportion of $\mathrm{B}$ was linearly increased to $25 \%$ in the next 6 min (14 to 20 $\min$ ) and then to $75 \% \mathrm{~B}$ in another $2 \mathrm{~min}$ (20 to $22 \mathrm{~min}$ ) and kept for $1 \mathrm{~min}$ (22 to $23 \mathrm{~min}$ ). Finally, the eluent composition was restored to the initial condition and maintained for 4 min for column conditioning (23 to $27 \mathrm{~min}$ ). The mobile phase consisted of (A) acetonitrile-water (20:80) containing $10 \mathrm{mM}$ ammonium acetate and (B) acetonitrile-water (80:20) containing 10 $\mathrm{mM}$ ammonium acetate. The flow rate was set at $0.4 \mathrm{~mL} / \mathrm{min}$. The MS analysis was performed using an LCMS-8060 mass spectrometer equipped with negative electrospray ionization mode working in the multiple reaction monitoring mode. All bile acids were determined with authentic chemicals, and quantification was performed with a standard curve. 


\section{Culture of primary hepatocytes}

Primary hepatocytes were prepared as previously reported [19]. In corticosterone treatment, after starvation with FBS-negative Williams' Medium E (Thermo Fisher Scientific, Waltham, MA, USA) for 2 hours, the hepatocytes were exposed to $0.1 \%$ dimethyl sulfoxide as vehicle and 30-1000 nM corticosterone. Six hours later, the cells were collected and subjected to quantitative PCR and western blot analysis. For lipid accumulation, the hepatocytes were exposed to $0.2 \%$ isopropanol/1\% ethanol/1\% FBS solution as vehicle, $100 \mu \mathrm{M}$ palmitic acid, or $100 \mu \mathrm{M}$ palmitic acid and $100 \mu \mathrm{M}$ bile acids ( $\beta \mathrm{MCA}$ or $\mathrm{CA}$ ). Forty-eight hours later, the cells were collected and subjected to quantitative PCR and western blot analysis. Another cell set was subjected to Oil Red O staining. Oil Red O staining was performed using 60\% isopropanol solution with Oil Red O dye. Then, haematoxylin and eosin (HE) staining was carried out with Mayer's haematoxylin solution (Muto Pure Chemicals Co., Tokyo, Japan) and pure eosin solution (Muto Pure Chemicals). For quantification of lipid accumulation, after Oil Red O staining, the dye was extracted from the stained cells with $40 \mathrm{mM} \mathrm{HCl/isopropanol}$ solution. The dye concentration was determined by measuring the absorbance at $500 \mathrm{~nm}$ and used as the relative lipid amount.

\section{Quantitative PCR}

RNA was extracted using TRIzol reagent (Thermo Fisher Scientific), and quantitative PCR analysis was performed using cDNA generated from $1 \mu \mathrm{g}$ of total RNA with a Superscript II or III Reverse Transcriptase kit and random oligonucleotides (Thermo Fisher Scientific). All primer sequences are listed in Supplementary Table 3. Quantitative PCRs were carried out using SYBR green PCR master mix (Thermo Fisher Scientific) in an ABI Prism 7500 System. Values were quantified using the comparative CT method, and samples were normalized to 18S ribosomal RNA. 


\section{Western blot analysis}

Mouse primary hepatocytes were lysed with sample buffer (100 mM Tris-HCl, pH 6.8, 20\% glycerol, 4\% sodium dodecyl sulphate, 10\% 2-mercaptoethanol) and subjected to western blotting. In experiments with liver tissue, the liver was homogenized with RIPA buffer ( $50 \mathrm{mM}$ Tris- $\mathrm{HCl}, \mathrm{pH} 8.0,150 \mathrm{mM} \mathrm{NaCl}, 1 \%$ Triton X-100, 1\% SDS) and centrifuged at 10,000xg for $10 \mathrm{~min}$. The supernatant (50 $\mu \mathrm{g}$ of protein) was subjected to western blotting. The samples were boiled for $3 \mathrm{~min}$ and then separated and transferred to Immobilon-P-membranes (PVDF membrane) using standard western blotting techniques. The membranes were incubated with an antibody against cytochrome P450 family (CYP) 7A1 at a dilution of 1:1,000 (ab52903, Santa Cruz Biotechnology, Santa Cruz, CA, USA). The signals were normalized to signals obtained with a GAPDH antibody (MAB374) used at a dilution of 1:10,000.

\section{Histological analysis}

Small blocks of liver tissue were frozen in Optimal Cutting Temperature compound after substitution of sucrose (using 5,10 and $30 \%$ sucrose substitution at $\left.4^{\circ} \mathrm{C}\right)$. Sections $(8-\mu \mathrm{m}$ thick) were fixed with formaldehyde neutral buffer solution and stained with Oil Red O and HE.

\section{Statistical analysis}

Statistical analysis was performed using Prism ${ }^{\circledR}$ version 6.0 (GraphPad Software Inc., San Diego, CA). A p-value of less than 0.05 was considered a significant difference. In the case of the mouse model, significance was indicated for the MCS-Non-stress vs the MCD-Non-stress (\#), the MCD-Non-stress vs the MCD-Stress (*) and the MCS-Non-stress vs the MCS-Stress $(\$)$. 


\section{Results}

A correlation between serum cortisol and bile acid levels was observed in both patients with NAFLD and NASH mice.

To investigate whether stress is associated with NAFLD, serum cortisol (a representative factor secreted by stress in humans) and BA levels of patients with NAFLD were measured. The serum cortisol level seemed to be significantly associated with the BA levels (Fig. 1A). In this study, the influence of restraint stress (stress) on steatohepatitis was investigated with a non-alcoholic steatohepatitis model [methionine- and choline-deficient (MCD) diet-fed mice]. Three weeks of continuous stress with MCD was carried out after pre-treatment with the MCD diet for 1 week (Fig. 1B). Stress did not affect the decreased ratio of body weight and relative liver mass under the condition of the MCD diet (Figs. 1B and 1C). In addition, stress did not change serum ALT levels (Fig. 1D) and expression levels of inflammatory cytokine genes in the livers (Fig. S1A). Interestingly, a correlation between serum corticosterone (a representative factor secreted by stress in rodents) and BA levels was observed in the mice as well as in the patients with NAFLD (Fig. 1E).

\section{Restraint stress enhanced hepatic and serum BA levels in NASH mice.}

Next, hepatic and serum BA levels were measured to investigate whether the correlation between corticosterone and BA in serum results from stress challenge. Under the MCD diet, stress significantly increased both serum and hepatic BA levels [23.6 to $38.8 \mu \mathrm{M}$ (1.64-fold) and 0.399 to $0.673 \mu \mathrm{mol} / \mathrm{g}$ liver (1.69-fold), respectively] (Fig. 2A). The stress trended to elevate hepatic BA levels under conditions of the MCS diet [the control and stress were 0.026 and $0.137 \mu \mathrm{mol} / \mathrm{g}$ liver (5.26-fold), respectively] (Fig. 2A). In addition, the hepatic BA 
composition was investigated. Hepatic tauro-beta-muricholic acid (TßMCA) and taurocholic acid (TCA) levels were significantly increased in MCD-induced NASH. However, these taurine-conjugated BAs were not changed after challenge with stress (Fig. 2B). Hepatic taurochenodeoxycholic acid (TCDCA) and taurodeoxycholic acid (TDCA) levels were not changed in MCD-induced NASH (Fig. 2B). Interestingly, non-conjugated BA and betamuricholic acid ( $\beta \mathrm{MCA})$ were significantly elevated after challenge with stress [1.14 to 3.46 nmol/g liver (3.03-fold)], and cholic acid (CA) tended to be elevated [0.84 to $2.24 \mathrm{nmol} / \mathrm{g}$ liver (2.67-fold)] (Fig. 2B). In the tested BA-related genes, the MCD diet increased hepatic expression levels of cytochrome P450 family (CYP) 7A1 (CYP7A1), solute carrier family (SLC) 51 beta (SLC51B, also called organic solute transporter subunit beta), and ATP-binding cassette (ABC) C4 genes (3.56-, 11.73-, and 7.09-fold, respectively) (Fig. 2C). Under the MCD diet, stress enhanced SCL51B and ABCC4 expression (11.73- to 15.55-fold and 7.09- to 14.47fold, respectively) (Fig. 2C). Interestingly, hepatic CYP7A1 expression was induced by stress under the condition of the MCS diet (4.03-fold). The results may suggest that stress directly stimulates hepatic Cyp7al induction. Hepatic expression of the other BA-related factors, namely, hepatic nuclear factor 4 alpha (HNF4A), liver rich homologue 1 (LRH1), farnesoid X receptor (FXR), small heterodimer partner (SHP), CYP7B1, CYP8B1, CYP27A1, bile acidCoA:amino acid N-acyltransferase (BAAT), ABCB11, ABCC2, SLC51A, ABCC3, ABCC5, SLC10A1, and solute carrier organic anion 1a1 (SLCO1A1), was not changed in either the MCS or MCD group after stress (Fig. 2C). The highest hepatic CYP7A1 protein level was observed in the MCD-stress group (Fig. 2D). Interestingly, both serum BA and corticosterone levels were positively correlated with hepatic CYP7A1 protein levels but not hepatic CYP7A1 mRNA levels (Fig. 2E). These results suggested the existence of the corticosterone-CYP7A1 protein-BA cascade in NASH livers after stress challenge. 


\section{Stress-stimulated corticosterone enhanced hepatic CYP7A1 protein levels.}

We investigated whether stress directly induced hepatic expression of the Cyp7a1, Slc51b and $A b c c 4$ genes. In mice with elevated serum corticosterone levels after 2 days of continuous stress (short stress) with a standard chow diet (Fig. 3A), an increasing tendency of hepatic CYP7A1 mRNA levels was observed (2.38-fold), while SLC51B and ABCC4 mRNA levels were not elevated (Fig. 3B). Furthermore, the CYP7A1 protein was significantly increased 1.39-fold after short stress (Fig. 3C). Next, we investigated whether corticosterone can induce Cyp7al gene expression in mouse primary hepatocytes. Increases in both CYP7A1 mRNA and protein levels were observed at 6 hours after corticosterone exposure (Fig. 3D). When the dosedependency of corticosterone in Cyp7al induction was investigated, the CYP7A1 protein level was increased in a dose-dependent manner, while the CYP7A1 mRNA level was not increased in a dose-dependent manner, although $300 \mathrm{nM}$ corticosterone increased CYP7A1 mRNA levels (Fig. 3E).

Restraint stress decreased hepatic lipid levels in NASH mice via elevated hepatic BMCA levels.

Oil Red O staining was performed to investigate whether stress can influence hepatic lipid homeostasis in the development of NASH. Oil Red O staining showed smaller lipid droplet sizes in the livers of the MCD-stress group than in the livers of the MCD-non-stress group (Fig. 4A). In addition, triglyceride (TG), total cholesterol (TChol) and non-esterified fatty acid (NEFA) levels were measured in serum and liver after stress challenge. Stress significantly decreased both hepatic TG and NEFA levels [624 to $353 \mathrm{mg} / \mathrm{g}$ liver (0.57-fold) and 0.336 to 
$0.242 \mathrm{Eq} / \mathrm{g}$ liver (0.72-fold), respectively] in the MCD group (Fig. 4B) without affecting fatty acid-related gene expression in the liver (Fig. 4C). However, stress had no effect on hepatic TChol (Fig. 4B) and cholesterol-related gene expression levels in the liver (Fig. S1B). Interestingly, $\beta \mathrm{MCA}$ inhibited lipid accumulation in primary-cultured mouse hepatocytes after exposure to palmitic acid (PA)/oleic acid (OA), while CA did not (Figs. 5A and 5B). However, the expression levels of lipid-related genes were not altered in the hepatocytes after treatment with $\beta$ MCA (Fig. 5C). On the other hand, the PA/OA-decreased CYP7A1 mRNA and protein levels tended to recover after treatment with $\beta \mathrm{MCA}$ (Figs. 5D and 5E). These results suggest that $\beta M C A$ can suppress hepatic lipid accumulation in NASH mice. 


\section{Discussion}

The current study demonstrated that stress increased serum BA levels in NASH mice with induction of Cyp7al, Slc51b and Abcc4 gene expression. The BA amount and composition are well known to be changed in serum in humans and rodents with NASH [20-22] . In addition, it has been suggested that $S L C 51 B$ and $A B C C 4$ gene expression (BA transporters from hepatocytes to blood $[23,24])$ is induced following NASH progression. Thus, the stresselevated serum BA levels may result from the stress-increased SLC51B and ABCC4 mRNA levels in NASH. However, the elevated SLC51B and ABCC4 mRNA levels could be regarded as a compensatory change, mainly because short stress did not induce $S l c 51 b$ and Abcc4 gene expression. Interestingly, unlike the $S l c 51 b$ and $A b c c 4$ genes, Cyp $7 a 1$ gene expression was induced under both MCS and MCD diet conditions after the stress challenge. In addition, the rodent stress marker corticosterone increased both CYP7A1 mRNA and protein levels in mouse primary hepatocytes. These results suggest that stress influences CYP7A1 expression in the liver via glucocorticoids. On the other hand, unlike the results of this study using male mice, it has been reported that stress did not change hepatic Cyp $7 a 1$ gene expression in female mice [25]. As oestrogen is involved in BA homeostasis through cholesterol metabolism [26, 27], this discrepancy may result from sex differences. Corticosterone is known to be a glucocorticoid receptor activator [28]. A report noted that the peak hepatic CYP7A1 mRNA level coincides with that of serum corticosterone levels in mice exhibiting a diurnal rhythm [29]. On the other hand, some reports noted that CYP7A1 mRNA levels were decreased in mice treated with the synthetic glucocorticoid receptor activators prednisolone and methylprednisolone $[30,31]$. The conflicting reports indicate the possibility that corticosterone activates factors other than synthetic glucocorticoid receptor activators. In the current study, the hepatic CYP7A1 protein level was correlated with the serum BA level rather than the 
CYP7A1 mRNA level. In mouse primary hepatocytes, the CYP7A1 protein level did not necessarily match the CYP7A1 mRNA level. Considering that the half-life of CYP7A1 mRNA is very short $[32,33]$, CYP7A1 protein should be used rather than CYP7A1 mRNA to understand the pathophysiology. A detailed study will be needed to further understand the molecular mechanism.

In humans, NASH has been reported to elevate glycol-CA and TCA levels [34-36] and reduce the ratio of secondary BAs/primary BAs [36]. In the present study, the MCD diet decreased the ratio of TCDCA and TDCA levels/T $\beta$ MCA and TCA levels in the mouse livers. As the hydrophilicity of BA is in the order conjugated BAs > unconjugated BAs and BMCA > $\mathrm{CA}>\mathrm{CDCA}>\mathrm{DCA}[37]$, these results may suggest that hydrophilic BA levels are upregulated in NASH. In addition, stress increased hepatic $\beta \mathrm{MCA}$ and CA levels in NASH without elevated hepatic T $\beta M C A$ and TCA levels. Furthermore, stress did not alter the expression of the Baat gene, which regulates the taurine conjugation of BA. Although the deconjugation reaction by gut flora needs to be considered, the observation supported the possibility that the stressincreased hepatic $\beta \mathrm{MCA}$ and CA levels result from the induction of Cyp $7 a 1$ gene expression, which is the rate-limiting enzyme of BA synthesis. FXR is a nuclear BA receptor that is strongly activated by CDCA and induces $S h p$ expression to suppress Cyp $7 a 1$ gene expression [38]. Although $\beta$ MCA can negatively act on FXR activation [39], changes in SHP expression levels were not observed in NASH livers after stress challenge. In addition, the Hnf4a and Lrhl gene expression levels, which upregulate $C y p 7 a 1$ gene expression, were not altered after the stress challenge. These results suggest that the stress induction of Cyp $7 a 1$ gene expression is mediated by other factors. Stress could contribute to the pathology changes in NASH through an increase in unconjugated BA levels. 
In contrast to this study in which serum TG and NEFA levels were not changed in B6 mice after the stress challenge, it has been reported that stress downregulates plasma TG and NEFA levels in normal mice (SV129 strain, NIH-31 diet) following upregulated fatty acid-related genes [40]. The discrepancy may result from the difference in mouse strain and diet. Generally, serum lipid levels are also regulated by adipose tissue in addition to the liver [41]. In addition, stress stimulates noradrenaline secretion via sympathetic nerve function [14] and can promote fat degradation by activating adrenergic receptor $\beta 3$ in adipose tissue [42]. The MCD diet is known to accelerate fat degradation in adipose tissue with a decline in body weight [43]. Thus, under conditions of an MCD diet, the effect of stress in adipose tissue might be hard to observe. Future studies will be needed to understand the molecular mechanism by which stress decreases hepatic lipid levels. Interestingly, the current study demonstrated that stress reduced hepatic TG and NEFA levels and decreased lipid droplet size in MCD-induced NASH mice with enhanced CYP7A1 expression. Cyp7al-transgenic mice have been reported to inhibit high-fat diet-induced fatty liver formation[44, 45], indicating that enhanced CYP7A1 expression is associated with the suppression of hepatic lipid accumulation. However, the molecular mechanism is still unclear. CYP7A1 is the rate-limiting enzyme for BA de novo synthesis [46, 47]. In this study, under conditions of MCD-induced NASH, stress elevated hepatic $\beta \mathrm{MCA}$ levels with enhanced CYP7A1 expression. Furthermore, treatment with $\beta$ MCA protected against PA/OA-induced lipid accumulation in hepatocytes. Thus, the present study provides a view that CYP7A1-produced $\beta M C A$ contributes to the suppression of lipid accumulation in hepatocytes, but it is still unknown how $\beta$ MCA suppresses hepatic lipid accumulation. Since $\beta \mathrm{MCA}$ is murine $\mathrm{BA}$ and is not synthesized in humans, these phenomena could not be observed in human patients with NAFLD. However, the results may suggest that $\beta$ MCA is available for NAFLD therapy. 
In clinical research, it is difficult to assess the effect of stress on diseases mainly because no index defines stress. In this study, the serum glucocorticoid level was used as the stress index. However, serum glucocorticoid levels can change due to various factors as well as stress. The results in this study may not necessarily match the clinical data but provide the possibility that stress can decrease hepatic lipid levels in patients with NAFLD. In the future, a stress index should be required to further understand the molecular mechanism by which stress influences our bodies.

In conclusion, the present study demonstrated that stress influences hepatic BA and lipid homeostasis in NASH mice, indicating the possibility that $\beta$ MCA is available for NASH therapy. It is expected that detailed molecular mechanisms will be elucidated to develop a NAFLD therapeutic drug. 


\section{Acknowledgements}

We thank Tadashi Mizutani, Kenji Kitamura, Chiho Kanodo, Kanako Fukuda (Osaka City University Graduate School of Medicine) and the Research Support Platform of Osaka City University Graduate School of Medicine for technical assistance. This work was supported by Osaka City University Strategic Research Grants 2012 and 2013 for young researchers (to T.M.), Takeda Science Foundation (to T.M.), JSPS KAKENHI Grant Numbers JP26870501 and 17K18012 (to T.M.), and Gilead Sciences (to H.F. and N.K.).

\section{Disclosures}

No conflicts of interest, financial or otherwise, are declared by the authors. 


\section{References}

1. Yuan L, Bambha K. Bile acid receptors and nonalcoholic fatty liver disease. World J Hepatol 2015;7:2811-8.

2. Michelotti GA, Machado MV, Diehl AM. NAFLD, NASH and liver cancer. Nat Rev Gastroenterol Hepatol 2013;10:656-65.

3. Younossi ZM, Koenig AB, Abdelatif D, Fazel Y, Henry L, Wymer M. Global epidemiology of nonalcoholic fatty liver disease-Meta-analytic assessment of prevalence, incidence, and outcomes. Hepatology 2016;64:73-84.

4. Fan JG, Zhu J, Li XJ, Chen L, Lu YS, Li L, et al. Fatty liver and the metabolic syndrome among Shanghai adults. J Gastroenterol Hepatol 2005;20:1825-32.

5. Wong VW, Wong GL, Choi PC, Chan AW, Li MK, Chan HY, et al. Disease progression of non-alcoholic fatty liver disease: a prospective study with paired liver biopsies at 3 years. Gut 2010;59:969-74.

6. Loomba R, Sanyal AJ. The global NAFLD epidemic. Nat Rev Gastroenterol Hepatol 2013;10:686-90.

7. Marengo A, Jouness RI, Bugianesi E. Progression and Natural History of Nonalcoholic Fatty Liver Disease in Adults. Clin Liver Dis 2016;20:313-24.

8. Browning JD, Horton JD. Molecular mediators of hepatic steatosis and liver injury. J Clin Invest 2004;114:147-52.

9. Neuschwander-Tetri BA. Hepatic lipotoxicity and the pathogenesis of nonalcoholic steatohepatitis: the central role of nontriglyceride fatty acid metabolites. Hepatology 2010;52:774-88.

10. Tilg H, Moschen AR. Evolution of inflammation in nonalcoholic fatty liver disease: the multiple parallel hits hypothesis. Hepatology 2010;52:1836-46.

11. Day CP. From fat to inflammation. Gastroenterology 2006;130:207-10.

12. Tanaka N, Matsubara T, Krausz KW, Patterson AD, Gonzalez FJ. Disruption of phospholipid and bile acid homeostasis in mice with nonalcoholic steatohepatitis. Hepatology 2012;56:118-29. 
13. van de Wouw M, Boehme M, Lyte JM, Wiley N, Strain C, O'Sullivan O, et al. Shortchain fatty acids: microbial metabolites that alleviate stress-induced brain-gut axis alterations. J Physiol 2018;596:4923-44.

14. Rosmond R, Dallman MF, Bjorntorp P. Stress-related cortisol secretion in men: relationships with abdominal obesity and endocrine, metabolic and hemodynamic abnormalities. J Clin Endocrinol Metab 1998;83:1853-9.

15. Russell AL, Tasker JG, Lucion AB, Fiedler J, Munhoz CD, Wu TJ, et al. Factors promoting vulnerability to dysregulated stress reactivity and stress-related disease. $\mathbf{J}$ Neuroendocrinol 2018;30:e12641.

16. Nagano J, Nagase S, Sudo N, Kubo C. Psychosocial stress, personality, and the severity of chronic hepatitis C. Psychosomatics 2004;45:100-6.

17. Marsland AL, Cohen S, Rabin BS, Manuck SB. Associations between stress, trait negative affect, acute immune reactivity, and antibody response to hepatitis B injection in healthy young adults. Health Psychol 2001;20:4-11.

18. Hagio M, Matsumoto M, Fukushima M, Hara H, Ishizuka S. Improved analysis of bile acids in tissues and intestinal contents of rats using LC/ESI-MS. J Lipid Res 2009;50:173-80.

19. Matsubara T, Tanaka N, Patterson AD, Cho JY, Krausz KW, Gonzalez FJ. Lithocholic acid disrupts phospholipid and sphingolipid homeostasis leading to cholestasis in mice. Hepatology 2011;53:1282-93.

20. Aranha MM, Cortez-Pinto H, Costa A, da Silva IB, Camilo ME, de Moura MC, et al. Bile acid levels are increased in the liver of patients with steatohepatitis. Eur $\mathbf{J}$ Gastroenterol Hepatol 2008;20:519-25.

21. Ferslew BC, Xie G, Johnston CK, Su M, Stewart PW, Jia W, et al. Altered Bile Acid Metabolome in Patients with Nonalcoholic Steatohepatitis. Dig Dis Sci 2015;60:331828.

22. Suga T, Yamaguchi H, Ogura J, Shoji S, Maekawa M, Mano N. Altered bile acid composition and disposition in a mouse model of non-alcoholic steatohepatitis. Toxicol Appl Pharmacol 2019;379:114664-. 
23. Dawson PA, Hubbert M, Haywood J, Craddock AL, Zerangue N, Christian WV, et al. The heteromeric organic solute transporter alpha-beta, Ostalpha-Ostbeta, is an ileal basolateral bile acid transporter. The Journal of biological chemistry 2005;280:6960-8.

24. Rius M, Hummel-Eisenbeiss J, Hofmann AF, Keppler D. Substrate specificity of human ABCC4 (MRP4)-mediated cotransport of bile acids and reduced glutathione. American journal of physiology Gastrointestinal and liver physiology 2006;290:G640G9.

25. Silvennoinen R, Quesada H, Kareinen I, Julve J, Kaipiainen L, Gylling H, et al. Chronic intermittent psychological stress promotes macrophage reverse cholesterol transport by impairing bile acid absorption in mice. Physiological reports 2015;3.

26. Lavoie J-M. Dynamics of hepatic and intestinal cholesterol and bile acid pathways: The impact of the animal model of estrogen deficiency and exercise training. World $\mathbf{J}$ Hepatol 2016;8:961-75.

27. Liu X, Xue R, Yang C, Gu J, Chen S, Zhang S. Cholestasis-induced bile acid elevates estrogen level via farnesoid $\mathrm{X}$ receptor-mediated suppression of the estrogen sulfotransferase SULT1E1. J Biol Chem 2018;293:12759-69.

28. Adcock IM, Mumby S. Glucocorticoids. Handb Exp Pharmacol 2017;237:171-96.

29. Mitropoulos KA, Balasubramaniam S. The role of glucocorticoids in the regulation of the diurnal rhythm of hepatic beta-hydroxy-beta-methylglutaryl-coenzyme A reductase and cholesterol 7 alpha-hydroxylase. Biochem J 1976;160:49-55.

30. Out C, Dikkers A, Laskewitz A, Boverhof R, van der Ley C, Kema IP, et al. Prednisolone increases enterohepatic cycling of bile acids by induction of Asbt and promotes reverse cholesterol transport. J Hepatol 2014;61:351-7.

31. Xiao Y, Yan W, Zhou K, Cao Y, Cai W. Glucocorticoid treatment alters systemic bile acid homeostasis by regulating the biosynthesis and transport of bile salts. Dig Liver Dis 2016;48:771-9.

32. Li T, Chanda D, Zhang Y, Choi HS, Chiang JY. Glucose stimulates cholesterol 7alphahydroxylase gene transcription in human hepatocytes. J Lipid Res 2010;51:832-42.

33. Li T, Kong X, Owsley E, Ellis E, Strom S, Chiang JY. Insulin regulation of cholesterol 7alpha-hydroxylase expression in human hepatocytes: roles of forkhead box $\mathrm{O} 1$ and sterol regulatory element-binding protein 1c. J Biol Chem 2006;281:28745-54. 
34. Kalhan SC, Guo L, Edmison J, Dasarathy S, McCullough AJ, Hanson RW, et al. Plasma metabolomic profile in nonalcoholic fatty liver disease. Metabolism 2011;60:404-13.

35. Lake AD, Novak P, Shipkova P, Aranibar N, Robertson D, Reily MD, et al. Decreased hepatotoxic bile acid composition and altered synthesis in progressive human nonalcoholic fatty liver disease. Toxicol Appl Pharmacol 2013;268:132-40.

36. Puri P, Daita K, Joyce A, Mirshahi F, Santhekadur PK, Cazanave S, et al. The presence and severity of nonalcoholic steatohepatitis is associated with specific changes in circulating bile acids. Hepatology (Baltimore, Md) 2018;67:534-48.

37. Attili AF, Angelico M, Cantafora A, Alvaro D, Capocaccia L. Bile acid-induced liver toxicity: relation to the hydrophobic-hydrophilic balance of bile acids. Med Hypotheses 1986;19:57-69.

38. Goodwin B, Jones SA, Price RR, Watson MA, McKee DD, Moore LB, et al. A regulatory cascade of the nuclear receptors FXR, SHP-1, and LRH-1 represses bile acid biosynthesis. Mol Cell 2000;6:517-26.

39. Sayin SI, Wahlström A, Felin J, Jäntti S, Marschall HU, Bamberg K, et al. Gut microbiota regulates bile acid metabolism by reducing the levels of tauro-betamuricholic acid, a naturally occurring FXR antagonist. Cell Metab 2013;17:225-35.

40. Konstandi M, Shah YM, Matsubara T, Gonzalez FJ. Role of PPARalpha and HNF4alpha in stress-mediated alterations in lipid homeostasis. PLoS One 2013;8:e70675.

41. Tanaka N, Takahashi S, Matsubara T, Jiang C, Sakamoto W, Chanturiya T, et al. Adipocyte-specific disruption of fat-specific protein 27 causes hepatosteatosis and insulin resistance in high-fat diet-fed mice. J Biol Chem 2015;290:3092-105.

42. Emorine LJ, Marullo S, Briend-Sutren MM, Patey G, Tate K, Delavier-Klutchko C, et al. Molecular characterization of the human beta 3-adrenergic receptor. Science $1989 ; 245: 1118-21$.

43. Machado MV, Michelotti GA, Xie G, Almeida Pereira T, Boursier J, Bohnic B, et al. Mouse models of diet-induced nonalcoholic steatohepatitis reproduce the heterogeneity of the human disease. PLoS One 2015;10:e0127991. 
44. Li T, Owsley E, Matozel M, Hsu P, Novak CM, Chiang JY. Transgenic expression of cholesterol 7alpha-hydroxylase in the liver prevents high-fat diet-induced obesity and insulin resistance in mice. Hepatology 2010;52:678-90.

45. Qi Y, Jiang C, Cheng J, Krausz KW, Li T, Ferrell JM, et al. Bile acid signaling in lipid metabolism: metabolomic and lipidomic analysis of lipid and bile acid markers linked to anti-obesity and anti-diabetes in mice. Biochim Biophys Acta 2015;1851:19-29.

46. Jelinek DF, Andersson S, Slaughter CA, Russell DW. Cloning and regulation of cholesterol 7 alpha-hydroxylase, the rate-limiting enzyme in bile acid biosynthesis. $\mathrm{J}$ Biol Chem 1990;265:8190-7.

47. Myant NB, Mitropoulos KA. Cholesterol 7 alpha-hydroxylase. J Lipid Res 1977; 18:135-53.

\section{Figure legends}

Figure 1. A correlation between serum cortisol and bile acid levels was observed in both patients with NAFLD and NASH mice.

(A) Correlation between serum cortisol and bile acid (BA) levels in patients with NAFLD. The correlation factor (r) and P-value were estimated with Pearson's correlation analysis $(n=30)$. (B-D) Changes in body weight (B), liver mass (C) and serum ALT level (D) after challenge with restraint stress. The methionine- and choline-supplemented diet, methionine- and cholinedeficient diet, restraint stress, and non-stressed control were expressed as MCS, MCD, Stress, and Non-stress, respectively. Data represent the mean and SD ( $n=5)$. Significance was determined by one-way analysis of variance (ANOVA) with Bonferroni's test (\#\#\#, $\mathrm{P}<0.001$ for the MCS-Non-stress vs the MCD-Non-stress). (E) Correlation between serum corticosterone and BA levels in the NASH mouse model. The correlation factor (r) and P-value were estimated with Pearson's correlation analysis $(n=20)$. 


\section{Figure 2. Restraint stress enhanced hepatic and serum BA levels in NASH mice.}

(A) Hepatic and serum BA levels after restraint stress challenge. Each bar represents the mean and SD ( $\mathrm{n}=5)$. Significance was determined by one-way ANOVA with Bonferroni's test (\#, $\mathrm{P}<0.05$; \#\#, $\mathrm{P}<0.01$ for the MCS-Non-stress vs the MCD-Non-stress. *, $\mathrm{P}<0.05$; **, $\mathrm{P}<0.01$ for the MCD-Non-stress vs the MCD-Stress group). (B) Hepatic BA concentration after challenge with stress. Each bar represents the mean and SD (n=5). Significance was determined by twoway ANOVA with Bonferroni's test (\#\#, $\mathrm{P}<0.01$ for the MCS-Non-stress vs the MCD-Nonstress. * $\mathrm{P}<0.05$ for the MCD-Non-stress vs the MCD-Stress group). $\mathrm{n}$ indicates no detectable level (less than $0.1 \mathrm{nmol} / \mathrm{g}$ liver). (C) Quantitative PCR analysis of BA-related gene expression. Quantitative PCR analysis of bile acid-related gene expression. Data are presented as the fold change relative to the value for the MCS-Non-stress group. Each bar represents the mean and SD ( $\mathrm{n}=5)$. Significance was determined by two-way ANOVA with Bonferroni's test (\#\#\#, $\mathrm{P}<0.001$ for the MCS-Non-stress vs the MCD-Non-stress. ***, $\mathrm{P}<0.001$ for the MCD-Nonstress vs the MCD-Stress group). (D) Western blot analysis of hepatic CYP7A1 protein after challenge with stress. (E) Correlation analysis among serum BA, corticosterone, and CYP7A1 mRNA and protein levels in the MCS and MCD diet-fed mice. The correlation factor (r) and P-value were estimated with Pearson's correlation analysis $(n=20)$.

Figure 3. Stress elevated hepatic CYP7A1 protein levels via corticosterone in mice.

(A) Serum corticosterone levels after challenge with short STR (short stress). Significance was determined by t-test. (***, P<0.001). (B) Quantitative PCR analysis of Cyp7a1, Slc51b, and Abcc4 gene expression. Data are presented as the fold change relative to the value for the nonstress group. (C) Western blot analysis of hepatic CYP7A1 protein after short stress challenge. 
The signal intensity was measured using ImageJ. Significance was determined by t-test. (**, $\mathrm{P}<0.01$ ). (D) Western blot analysis of CYP7A1 protein (6 and 24 hours) and quantitative PCR analysis for Cyp7al gene expression in mouse primary hepatocytes at 6 hours after treatment with $300 \mathrm{nM}$ corticosterone. Data are presented as the fold change relative to the value for the vehicle. Each bar represents the mean and SD $(n=3)$. Significance was determined by unpaired Student's t-test (**, $\mathrm{P}<0.01$ for the vehicle vs corticosterone). (E) Cyp7al gene expression analysis in mouse primary hepatocytes at 6 hours after treatment with corticosterone in a dosedependent manner. (Upper panel; western blot analysis, bottom panel; quantitative PCR analysis.)

\section{Figure 4. Influence of restraint stress on hepatic lipid levels in NASH mice.}

(A) Haematoxylin and eosin (HE) and Oil Red O staining for the livers after challenge with the stress and MCD diet. The central and portal veins are expressed as CV and PV, respectively. Bar represents $100 \mu \mathrm{m}$. (B) Hepatic and serum triglyceride (TG), total cholesterol (TChol), and non-esterified fatty acid (NEFA) in the mice. Data represent the mean and SD $(n=5)$. Significance was determined by one-way ANOVA with Bonferroni's test (\#\#\#, $\mathrm{P}<0.001$ for the MCS-Non-stress vs the MCD-Non-stress. *, P<0.05 for the MCD-Non-stress vs the MCDStress group). (C) Quantitative PCR analysis of fatty acid-related gene expression. Data are presented as the fold change relative to the value for the MCS-Non-stress group and represent the mean and SD $(n=5)$. Significance was determined by one-way ANOVA with Bonferroni's test (\#\#\#, $\mathrm{P}<0.001$ for the MCS-Non-stress vs the MCD-Non-stress).

Figure 5. $\beta M C A$ can inhibit lipid accumulation in mouse primary hepatocytes. 
(A and B) Oil Red $\mathrm{O}$ and haematoxylin staining in mouse primary hepatocytes after treatment with PA/OA and BA (A, $\beta$ MCA; B, CA). Bar represents $100 \mu \mathrm{m}$. Data are presented as the fold change relative to the value for the vehicle group and represent the mean and SD $(n=5)$. (C) Quantitative PCR analysis of fatty acid-related gene expression. Data are presented as the fold change relative to the value for the vehicle group and represent the mean and SD $(n=3)$. Significance was determined by one-way ANOVA with Bonferroni's test ${ }^{*}, \mathrm{P}<0.05$; **, $\mathrm{P}<0.01$; ***, $\mathrm{P}<0.001$ ). (D and E) Quantitative PCR and western blot analyses of Cyp7al gene expression in hepatocytes (D, quantitative PCR analysis; E, western blot analysis). Data are presented as the fold change relative to the value for the vehicle group and represent the mean and $\mathrm{SD}(\mathrm{n}=3)$. 